\title{
Automatisierte Reputationsverfolgung auf einem agentenbasierten elektronischen Marktplatz
}

\author{
Boris Padovan $^{\alpha}$, Stefan Sackmann ${ }^{\beta}$, Torsten Eymann ${ }^{\beta}$, Ingo \\ Pippow $^{\beta}$, Holger Eggs ${ }^{\beta}$ \\ ${ }^{\alpha}$ Atraxis AG, CH-8058 Zürich-Airport, \\ Tel. 0041-1-812 32 15, Fax 0041-1-812 19 57, \\ bpadovan@atraxis.com \\ ${ }^{\beta}$ Institut für Informatik und Gesellschaft, Abt. Telematik, \\ Albert-Ludwigs-Universität Freiburg, \\ Friedrichstraße 50, 79098 Freiburg im Breisgau, \\ Tel. 0761-203-4963, Fax 0761-203-4929, \\ \{ sackmann|eymann|pippow|eggs\}@iig.uni-freiburg.de
}




\title{
Automatisierte Reputationsverfolgung auf ein- em agentenbasierten elektronischen Marktplatz
}

\author{
Boris Padovan \\ Atraxis AG, CH-8058 Zürich-Airport, bpadovan@atraxis.com
}

Stefan Sackmann, Torsten Eymann, Ingo Pippow, Holger Eggs

Institut für Informatik und Gesellschaft, Abt. Telematik, Albert-Ludwigs-

Universität Freiburg, \{sackmann|eymann|pippow|eggs\}@iig.uni-freiburg.de

\begin{abstract}
Zusammenfassung: Software-Agenten werden zukünftig im digitalen Wirtschaften eine wichtige Rolle spielen. Ein kooperatives Verhalten der Agenten kann trotz Nutzung technischer Sicherheitsmechanismen, wie z.B. Kryptographie oder digitaler Signierung, jedoch nicht vorausgesetzt werden. Instanzen, die betrügerisches Verhalten in offenen Netzen sanktionieren, existieren bisher nur ansatzweise. In diesem Beitrag wird ein Reputationsmechanismus für agentenbasierte elektronische Märkte entwickelt, der das frühere Kooperationsverhalten agentenbasierter Marktteilnehmer erfasst, diese an andere Agenten weiterleitet und damit zukünftiges Verhalten beeinflusst. Der Mechanismus wurde prototypisch implementiert und es kann gezeigt werden, dass dessen Einsatz betrügerische Agenten von der Marktteilnahme in zunehmendem Maße ausschließt.
\end{abstract}

Schlüsselworte: Vertrauen, Reputationsmechanismus, Software-Agenten, MultiAgenten-System, Elektronische Marktplätze

\section{Vertrauen im Electronic Commerce}

\subsection{Software-Agenten in elektronischen Märkten}

Software-Agenten ${ }^{1}$ sind autonom handelnde Softwareobjekte, die in einer definierten Umgebung von ihrem Besitzer vorgegebene Ziele verfolgen [WoJe99].

\footnotetext{
In diesem Artikel werden die Begriffe „Agent“ bzw. „Software-Agent“ synonym verwendet und bezeichnen immer eine softwarebasierte Entität.
} 
Agenten müssen in einer sich permanent verändernden, dynamischen Umgebung zwischen Handlungsalternativen auswählen und dabei über adaptive Verfahren die Entscheidungsregeln der Aktionsauswahl ständig anpassen.

Ein elektronischer Markt wird von ,einem oder mehreren zusammenarbeitenden Informationssystemen [gebildet], die einzelne oder alle Phasen einer Markttransaktion elektronisch unterstützen, integrieren oder gar automatisieren“ [MüSc97]. Er ist geprägt vom parallelen Handeln einer Vielzahl menschlicher und softwarebasierter Teilnehmer. Agenten können eine Vielzahl vernetzter Märkte nicht nur permanent im Auftrag ihres Absenders beobachten, sondern dort auch Transaktionen initiieren. Marktteilnehmer werden sich daher zunehmend von autonomen Agenten auf den elektronischen Märkten repräsentieren lassen.

\subsection{Vertrauen und Reputation in elektronischen Märkten}

Vertrauensprobleme zählen zu den größten Hürden im Electronic Commerce [EgEn00]. Sie resultieren aus asymmetrisch verteilten transaktionsrelevanten Informationen [HiRi79] sowie aus der Möglichkeit opportunistischen Verhaltens [Will85] und beschränkter Rationalität [Simo57] der Akteure. Mehrseitig sichere Kommunikation [MüRa99] alleine liefert noch keine Gewähr dafür, dass sich Transaktionspartner, bzw. ihre Agenten, in einer wechselseitig vorhersagbaren Weise verhalten. Neben notwendiger technischer Sicherheit ist für eine weitergehende Nutzung der ökonomischen Potenziale elektronischer Märkte der Aufbau und die Aufrechterhaltung von Vertrauen ein zusätzliches, zur Sicherheit komplementäres Erfordernis [Eggs01].

\subsubsection{Vertrauen}

Vertrauen setzt sich aus einer Vertrauenshandlung und einer Vertrauenserwartung zusammen [Ripp98]:

\begin{tabular}{|l|l|}
\hline Vertrauen ist ... & $\begin{array}{l}\text {... die freiwillige Erbringung einer riskanten Vorleistung } \\
\text { unter Verzicht auf explizite rechtliche Sicherungs- und } \\
\text { Kontrollmaßnahmen gegen opportunistisches Verhalten ... }\end{array}$ \\
\hline Vertrauenshandlung & $\begin{array}{l}\ldots \text { in der Erwartung, dass der Vertrauensnehmer freiwillig } \\
\text { auf opportunistisches Verhalten verzichtet. }\end{array}$ \\
\hline Vertrauenserwartung
\end{tabular}

\subsubsection{Reputation}

Der Aufbau und die Aufrechterhaltung von Vertrauen kann durch verschiedene Bedingungen und Mechanismen erleichtert werden. Reputationsmechanismen, die auf bisheriges Verhalten der Transaktionspartner zurückgreifen, spielen eine entscheidende Rolle. Reputation wird dabei als eine Menge von Eigenschaften einge- 
führt, die einer Person (oder Unternehmen) aufgrund ihrer Historie zugeschrieben wird, und die als indikativ für ihr künftiges Verhalten betrachtet wird [Klei97]. Reputation wird in der Regel schrittweise, mit steigendem Risiko aufgebaut.

\subsection{Vertrauensunterstützende Institutionen}

Vertrauensunterstützende Institutionen helfen, vertrauensrelevante Informationen aufzudecken, zu bewerten und zu kommunizieren. Neben den Informationswirkungen, die aus dem Abbau asymmetrischer Informationen resultieren, machen vor allem Sanktionswirkungen den Mehrwert vertrauensunterstützender Institutionen aus. Durch die glaubwürdige Kommunikation von Fehlverhalten eines Akteurs oder Agenten wird dessen Reputation nicht nur innerhalb der ursprünglichen Transaktion gemindert, sondern innerhalb einer Gruppe potentieller künftiger Transaktionspartner. Somit steigen die Opportunitätskosten fehlerhaften Verhaltens. Vertrauensunterstützende Institutionen lassen sich, wie in Abbildung 1 dargestellt, transaktionsphasenabhängig strukturieren [Eggs01].

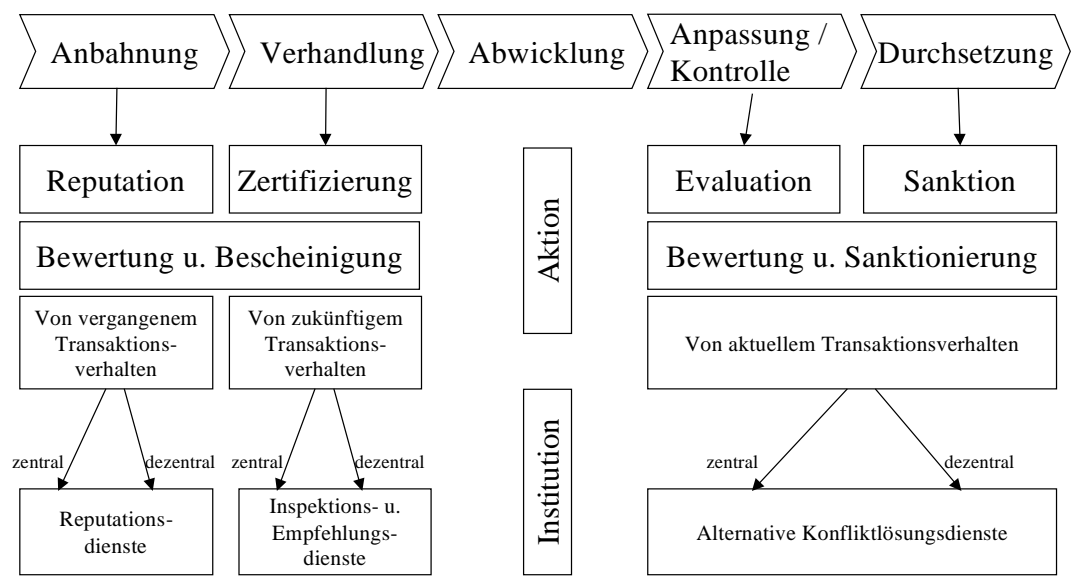

Abbildung 1: Transaktionsphasenabhängige Vertrauensgenese [Eggs01]

Reputationsdienste werden vor der Transaktion in Anspruch genommen und beziehen sich auf die Transaktionssubjekte. Sie erleichtern die Einschätzung der subjektiven Vertrauenswürdigkeit der Transaktionspartner, indem sie glaubhafte Aussagen über vergangenes Verhalten von Transaktionspartnern treffen. (zu Inspektions-, Empfehlungs- und Konfliktlösungsdiensten vgl. [Eggs01]

Im Folgenden wird ein Reputationsdienst für agentenbasierte Marktplätze vorgestellt, der früheres Kooperationsverhalten eines bestimmten Transaktionspartners in die Auswahl zukünftiger Transaktionspartner einbezieht. 


\section{Das Multi-Agenten-System AvalanCHE: Prototyp eines agentenbasierten elektronischen Marktplatzes}
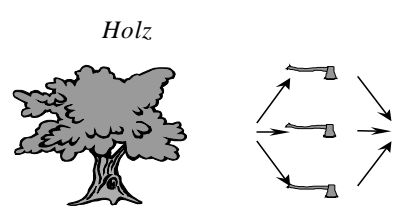

Bretter

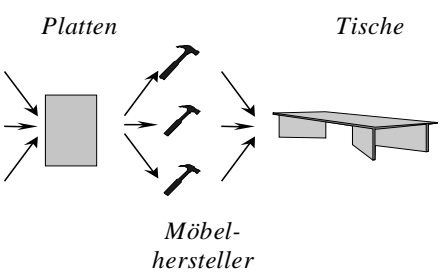

Abbildung 2: Modellhafte Wertschöpfungskette

Die Analyse zentraler und dezentraler Institutionen zum Vertrauensaufbau in elektronischen Märkten wird mit Hilfe des Multi-Agenten-Systems AVALANCHE vorgenommen, einem Prototyp eines offenen elektronischen Marktplatzes, auf dem menschliche Akteure durch Agenten repräsentiert werden. Die Agenten repräsentieren Unternehmen einer modellhaften Wertschöpfungskette der holzverarbeitenden Industrie (Holzfäller, Schreiner und Möbelhersteller, siehe Abbildung 2), die ihren Agenten ein quantitatives Verhandlungsziel sowie eine Verhandlungsstrategie vorgeben [Prei98]. Die Agenten sind automatisierte Produzenten [KeHa00], die auf elektronischen Marktplätzen benötigte Inputfaktoren von anderen Agenten kaufen, sie durch einen (virtuellen) Produktionsprozess in ein Zwischen- oder Endprodukt umwandeln und dieses an andere Agenten verkaufen. Eine detaillierte Beschreibung des Systems und seines technischen Aufbaus findet sich in [Eyma00].

\subsection{Automatisiertes Verhandeln zwischen Agenten}

Alle Agenten in AvalANCHE verfolgen das Ziel der Gewinnmaximierung, d.h. sie versuchen, Rohstoffe billig zu kaufen und hergestellte Güter teuer zu verkaufen. Jeder Agent entscheidet autonom und in Abhängigkeit von der aktuellen Marktsituation sowie den eigenen Finanz- und Lagerbeständen, welche Aktion als nächste verfolgt wird. Die „Strategie“ eines Agenten ergibt sich aus der Kombination numerischer Parameter, gegen die eine stochastische Probe durchgeführt wird, deren Ausgang die jeweils folgende Aktion bestimmt. Das Verhalten eines AVALANCHE-Agenten wird damit durch einen nicht-deterministischen endlichen Automaten bestimmt. Mit dieser Implementation ist es möglich, eine auf wenigen Parametern basierende adaptive Strategie einzusetzen, die einem komplexen deterministischen Regelwerk äquivalent ist [HoU194].

Die einzelnen Marktphasen werden wie folgt realisiert: In der Anbahnungsphase müssen Käufer und/oder Verkäufer potentielle Transaktionspartner auswählen. In der Verhandlungsphase startet der Käuferagent eine bilaterale Kommunikation mit 
dem Verkäuferagenten, indem er ihn zur Abgabe eines Preisgebots auffordert. Entsprechend einem „monotonic concession protocol“ [RoZ194] werden dann sequentiell Angebot und Gegenangebot ausgetauscht, bis es zu einer Einigung kommt oder die Verhandlung abgebrochen wird. In der Kontrollphase wird die Abwicklung der Transaktion von den beteiligten Agenten überwacht. Die beiden Transaktionspartner tauschen Geld gegen Ware. Erst in diesem Moment lässt sich feststellen, ob die Transaktion entsprechend der Vereinbarungen durchgeführt wurde und das Vertrauen in den Transaktionspartner berechtigt war.

\subsection{Referenzszenario: Kooperative Agenten in AVALANCHE}

Um das Verhalten des prototypischen elektronischen Marktplatzes AVALANCHE zu testen, wurden mehrere Testreihen durchgeführt, die je aus mehreren Testläufen bestehen, die unter gleichen Startbedingungen stets zu vergleichbaren Mustern führen. Die erste dargestellte Testreihe wird mit 50 homogenen Agenten jeden Typs durchgeführt, die bei jeder Transaktion kooperieren.

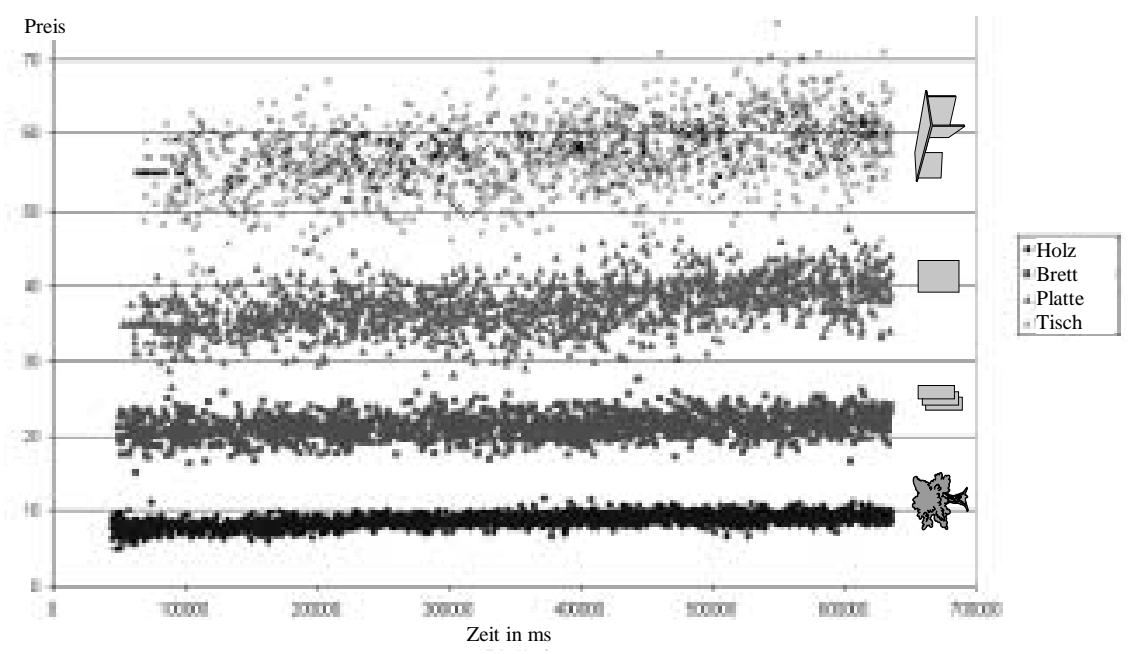

Abbildung 3: Preisentwicklung der gehandelten Güter

Exemplarisch ist in Abbildung 3 die Entwicklung der Güterpreise im Zeitablauf dargestellt, in der jeder Punkt eine abgeschlossene Transaktion markiert. Entlang der Ordinate ist die Wertschöpfungskette abgetragen. Da alle Agenten eine konstante identische Verhandlungsstrategie besitzen, ist es keinem Agenten möglich, sich einen preislichen Vorteil $^{2}$ herauszuarbeiten.

2 Die größere Varianz der Güterpreise mit zunehmender Wertschöpfungsstufe scheint eine Variante des „Peitscheneffektes“ zu sein [LePa97]. 
In einer zweiten Testreihe (nicht dargestellt) wird die wirtschaftliche Entwicklung der Agenten anhand ihres Vermögens ermittelt, wobei alle Parameter aus der ersten Testreihe beibehalten werden. Lediglich die Anzahl der Agenten je Typ wird auf drei beschränkt, um den Erfolg einzelner Agenten besser darstellen zu können. Die Ergebnisse beider Testreihen kommen unter der Voraussetzung zustande, dass sich alle Agenten stets kooperativ verhalten, d.h. Geld und Waren zu den vereinbarten Werten bzw. Mengen austauschen.

Die dritte Testreihe bildet ein offenes System nach, in dem Agenten ein betrügerisches Verhalten möglich ist. Die Parameterwahl entspricht der zweiten Testreihe, allerdings wird einer der Schreiner-Agenten als Betrüger gestartet, d.h. er liefert nach Abschluss einer Transaktion entweder die Ware nicht aus oder bezahlt nicht. Die beiden anderen Schreiner-Agenten hingegen verhalten sich stets kooperativ.

Abbildung 4 zeigt, dass der betrügende Agent sein Vermögen ausweiten kann, während das der kooperativen Agenten stagniert. Der betrügende Agent kann zudem den Großteil (57\%) der durchgeführten Transaktionen auf sich ziehen. Die Teilnahme des betrügenden Agenten führt zum Systemzusammenbruch, der im wirtschaftlichen Niedergang seiner vertikalen Transaktionspartner begründet liegt. Diese können aus den Transaktionen keine Gewinne realisieren und den Betrüger weder vor einer Transaktion erkennen noch nachträglich sanktionieren.

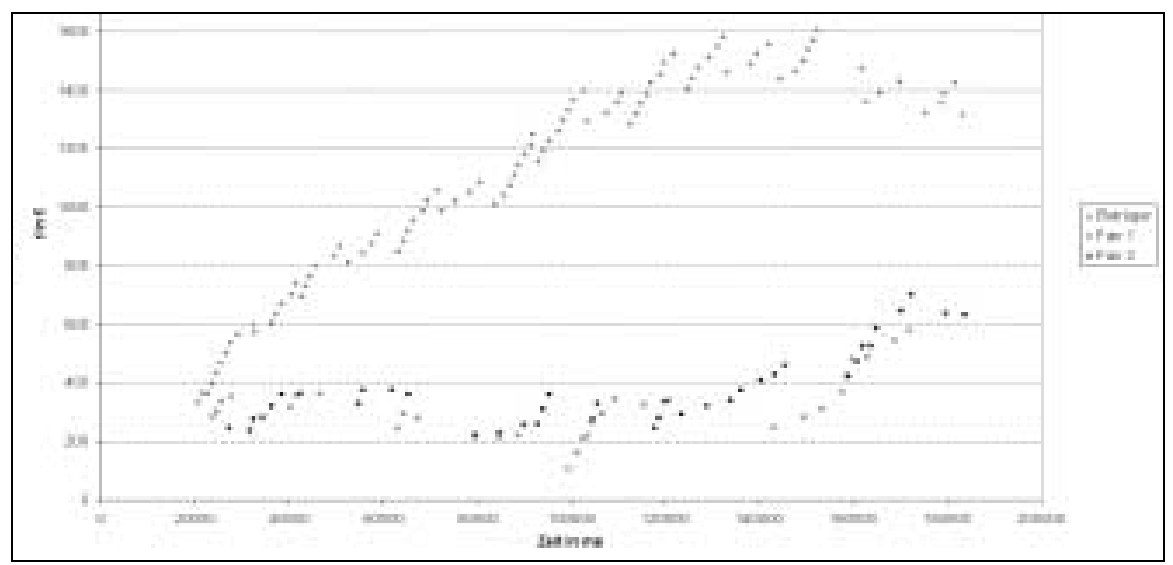

Abbildung 4: Vermögensentwicklung bei unkooperativem Verhalten eines Agenten

Der Vergleich zwischen der zweiten und dritten Testreihe zeigt, dass betrügerisches Verhalten einzelner Marktteilnehmer zu einem Versagen des Systems führen kann. Für offene agentenbasierte Marktplätze muss daher ein Mechanismus bereitgestellt werden, der betrügerische Agenten erkennt und sanktioniert. 


\section{Der Reputationsmechanismus}

\subsection{Modellierung von Reputation in Multi-Agenten-Systemen}

Existierende Modellierungsansätze zur Formalisierung von Reputation werden ausführlich in [Pado01] diskutiert. Alle angeführten Modelle beschränken sich auf die Schätzung und Darstellung von Reputationsparametern und überlassen die Verarbeitung den menschlichen Agentenbesitzern. Agenten können mit automatischen Verhandlungsmechanismen sich wiederholende Transaktionen autonom durchführen, jedoch müssen dann betrügerische Agenten ebenfalls automatisch sanktioniert werden können. Die untersuchten Ansätze sind u.E. für einen Einsatz in offenen, agentenbasierten elektronischen Marktplätzen nicht ausreichend, da sie Reputationsinformationen nicht weiterverarbeiten. AVALANCHE ist unseres Wissens das erste MAS, welches eine derartige Verarbeitung realisiert.

Reputation wird i.d.R. als numerische Variable auf einer Kardinal- oder Ordinalskala dargestellt. Dieser Wert fließt in den Auswahlprozess eines Transaktionspartners als Schwellwert oder Modifikator weiterer Entscheidungsparameter (z.B. Angebotspreis) ein. Die Reputation eines Agenten $X$ entsteht durch Informationen über seine vergangenen und die Erwartungen über seine zukünftige Aktionen. Sie kann durch eine Wahrscheinlichkeitsverteilung bzgl. der Verhaltensalternativen dargestellt werden. Informationen ergeben sich aus der Bewertung bisheriger Kooperationsbereitschaft durch frühere Transaktionspartner. „Gute“ Reputation bedeutet, dass diese zukünftig ein kooperatives Verhalten erwarten, während eine „schlechte“ Reputation ein unkooperatives Verhalten erwarten lässt.

\subsection{Formalisierung und Implementation}

\subsubsection{Reputationskonstante und Reputationskoeffizient}

Im einfachsten Fall, welcher der Implementation von AVALANCHE zugrunde liegt, bestehen nur zwei Verhaltensalternativen: Kooperation und Defektion. Nach erfolgreichem Abschluss einer Transaktion muss Geld gegen Ware getauscht werden. Ein Agent, der kooperiert, zahlt bzw. liefert nach Vereinbarung. Ein Agent, der defektiert, zahlt oder liefert nicht. Welches Verhalten ein Agent konkret wählen wird, kann von dem Transaktionspartner ex-ante nur geschätzt werden.

Eine strategische Entscheidung des Agenten bezüglich Kooperation oder Defektion wird entsprechend seinem konstanten Parameter reputation, der individuellen Reputationskonstante, getroffen. Dieser Wert liegt zwischen 0 und 1 und definiert die Eintrittswahrscheinlichkeit der Verhaltensalternative "Kooperation". Der Wert selbst ist für andere Agenten nicht einsehbar. 
Der Reputationskoeffizient dagegen ist ein Schätzwert eines anderen Agenten für die Reputationskonstante, welcher aus dem tatsächlichen Verhalten über mehrere Transaktionen hinweg angenähert wird. Jeder Agent führt prinzipiell für jeden möglichen Transaktionspartner einen eigenen Reputationskoeffizienten mit, der an andere Agenten weitergegeben werden kann. Der Reputationskoeffizient wird formal beschrieben durch:

$\mathrm{R}_{Y}^{X}$, wobei $\quad X$ die Identität des bewertenden Agenten darstellt und $Y$ die Identität des bewerteten Agenten. Der Reputationskoeffizient ist damit die Einschätzung der Wahrscheinlichkeit des zukünftigen Kooperationsverhaltens von $Y$ durch $X$ und liegt zwischen $0 \leq R_{Y}^{X} \leq 1$, wobei $R_{Y}^{X}=1$ eine gute und $R_{Y}^{X}=0$ eine schlechte Reputation darstellt.

\subsubsection{Ablauf des Reputationsmechanismus}

Jeder Reputationsmechanismus muss folgende fünf Aufgaben lösen [Pado01]:

1. Erfassung: Nach der Durchführung einer Transaktion wird das jeweilige Kooperationsverhalten des Partners erfasst.

2. Bewertung: Das erfasste Verhalten wird bewertet.

3. Speicherung: Die Bewertungen werden gespeichert. Je nach Implementation kann diese Speicherung bei einem der Beteiligten der Transaktion, aber auch bei einer dritten Partei stattfinden.

4. Abfrage: Vor der Durchführung einer neuen Transaktion werden die Bewertungen abgerufen und verarbeitet. Im vorliegenden Modell wird eine Entscheidungssituation über die Durchführung einer Verhandlung mit einem Agenten durch diese Informationen beeinflusst.

5. Anpassung: In dieser Phase kann der Agent aufgrund der erhaltenen Informationen die eigene Verhandlungsstrategie entsprechend modifizieren.

\subsubsection{Erfassung des Kooperationsverhaltens}

Nach Abschluss jeder Transaktion erfassen die beteiligten Agenten ihr Verhalten gegenseitig mit dem Faktor $r_{j}$ ( $j$ als Transaktionsindex). Im vorliegenden Modell gibt es nur die binären und objektiv mess- und bewertbaren Fälle „Ware/Geld erhalten (kooperiert)“ und „Ware/Geld nicht erhalten (defektiert)“. War eine Transaktion aufgrund betrügerischen Verhaltens eines Agenten nicht erfolgreich, erhält dieser eine Bewertung von $r_{j}=0$. Eine erfolgreiche Transaktion führt zu $r_{j}=1$.

\subsubsection{Bewertung und Speicherung des Kooperationsverhaltens}

Das erfasste Kooperationsverhalten $r_{j}$ führt zu einer Aktualisierung der Reputationskoeffizienten beider beteiligten Transaktionspartner. In AVALANCHE wird 
dafür ein exponentiell gewogener Mittelwert berechnet, der den zeitlichen Verlauf des Kooperationsverhaltens einbezieht. Damit ist der Mechanismus für Szenarien offen, in denen das Kooperationsverhalten der Agenten über die Zeit wechselt. Zur Berechnung des neuen Reputationskoeffizienten $\mathrm{R}_{Y j}^{\mathrm{X}}$ nach einer erfassten Transaktion wird die Abweichung zwischen dem erwarteten Kooperationsverhalten $\mathrm{R}_{Y j-1}^{\mathrm{X}}$ und dem tatsächlichen Kooperationsverhalten $\mathrm{r}_{j}$ mit einem Gewichtungsfaktor $\alpha$ berechnet. Der aktualisierte Reputationskoeffizient ergibt sich als:

$$
R_{Y j}^{X}=R_{Y j-1}^{X}+\alpha \cdot\left(r_{j}-R_{Y j-1}^{X}\right)
$$

Wird der Reputationskoeffizient nicht bei den Agenten selbst, sondern in einer speziellen Rating Agentur bewertet, so berücksichtigt diese auch die Vertrauenswürdigkeit der Bewertungsinformation der beteiligten Agenten $\mathrm{r}_{j}^{\mathrm{V}}(\mathrm{V}=\mathrm{X}, \mathrm{Y})$. Entsprechend berechnen sich die Reputationskoeffizienten dann wie folgt:

$$
\begin{aligned}
& R_{X j}=R_{X j-1}+\beta \cdot\left(r_{j}^{Y}-R_{X j-1}\right) \text { mit } \beta=\alpha \cdot R_{Y j-1} \quad \text {, bzw. }^{3} \\
& R_{Y j}=R_{Y j-1}+\beta \cdot\left(r_{j}^{X}-R_{Y j-1}\right) \text { mit } \beta=\alpha \cdot R_{X j-1}
\end{aligned}
$$

wobei der Gewichtungsfaktor $\beta$ und damit die Bedeutung der Gewichtung von der Reputation des bewertenden Agenten abhängt. Implizit wird hiermit die Annahme getroffen, dass das Kooperationsverhalten und das Bewertungsverhalten der einzelnen Agenten identisch ist.

Für den speziellen Fall, dass vor der Transaktion keinerlei Informationen über den zu bewertenden Agenten existierte, ergibt sich folgende Bewertungsformel:

$$
R_{X 1}=R_{X 0}+\beta \cdot\left(r_{j}^{Y}-R_{X 0}\right) \text { mit } \beta=\alpha \cdot R_{Y j-1}
$$

[FrRe99] befürworten, dass Agenten durch minimale anfängliche Reputation, also $R_{X 0}=0$, davon abgehalten werden können, sich zunächst unkooperativ zu verhalten und anschließend die Identität zu wechseln. Ein Identitätswechsel ist für Agenten, die ihrem Prinzipal eindeutig zugeordnet werden können, jedoch nicht möglich. Daher wird im vorliegenden Modell der anfängliche Reputationskoeffizient zur Beschleunigung des Handelsprozesses auf Basis des arithmetischen Mittels $R_{X 0}=\bar{R}$ der bekannten Reputationskoeffizienten berechnet. Unbekannte Agenten erhalten somit einen Vertrauensvorschuss. Es ergibt sich für diesen Fall:

$$
R_{X 1}=\bar{R}+\beta \cdot\left(r_{j}^{Y}-\bar{R}\right) \text { mit } \beta=\alpha \cdot R_{X j-1}
$$

Die quasi spiegelbildliche Berechnung des Reputationskoeffizienten von Käufer und Verkäufer wird im restlichen Teil des Beitrags nicht weiter ausgeführt. 


\subsubsection{Abfrage von Reputationsinformationen}

Es gibt verschiedene Möglichkeiten, wie ein Agent $X$ Reputationsinformationen über einen anderen Agenten $Y$, mit dem eine Transaktion geplant ist, beziehen kann. Diese hängen von der Bekanntheit des Agenten $Y$ ab:

Fall 1: Es existieren keinerlei Reputationsinformationen über Agent $Y$ : der Agent $Y$ ist $X$ weder unmittelbar, noch über eine $X$ zugängliche dritte Instanz mittelbar bekannt. Der Reputationskoeffizient wird dann als Mittelwert aller ihm (oder der dritten Partei) bekannten Reputationskoeffizienten $R_{Y 0}^{X}=\bar{R}$ gebildet (s.o.).

Fall 2: Agent $Y$ ist zwar dem Agenten $X$ selbst unbekannt, jedoch über einer $X$ bekannten dritten Instanz mittelbar bekannt. In diesem Fall kann der Agent $X$ die von der dritten Instanz zur Verfügung gestellten Reputationsinformationen nutzen und entsprechend $R_{Y 0}^{X}=R_{Y}$ setzen.

Fall 3: Der Agent $X$ hat bereits Transaktionen mit $Y$ durchgeführt und kann daher seine eigenen Erfahrungen mit der Kooperativität von $Y$ zur Einschätzung dessen Kooperationsverhaltens nutzen. ${ }^{4}$

\subsubsection{Anpassung des Verhaltens an Reputationsinformationen}

In Abhängigkeit der zur Verfügung stehenden Reputationsinformationen können die Agenten ihr Verhalten anpassen. Bei AvALANCHE wirkt der Reputationskoeffizient als Modifikator auf den Angebotspreis. Zu Beginn einer Verhandlung erhält ein AvalANCHE-Agent Angebote von potentiellen Transaktionspartnern. Diese Angebote bewertet der Agent mit den zur Verfügung stehenden Reputationsinformationen, indem er zusätzlich zum geforderten Preis $p_{i}$ den Erwartungswert des Verlustes in seine Entscheidung mit einfließen lässt. Dazu berechnet der Agent einen modifizierten Angebotspreis entsprechend der Gleichung:

$$
p_{i}^{*}=p_{i}+\left(R_{Y i}^{X} \cdot 0+\left(1-R_{Y i}^{X}\right) \cdot p_{i}\right)=p_{i} \cdot\left(2-R_{Y i}^{X}\right)
$$

Dieser modifizierte Angebotspreis ist Grundlage für die Aufnahme einer bilateralen Verhandlung, sowie für die weitere Verhandlungsstrategie.

4 Eventuell kann Agent $X$ zusätzlich die Dienste einer dritten Instanz in Anspruch nehmen und deren Reputationsinformationen mit den eigenen Erfahrungen kombinieren, dieser Fall wird hier jedoch nicht betrachtet. 


\section{Experimentelle Ergebnisse unter Einsatz des Reputationsmechanismus}

Die in AvAlANCHE erzielten Ergebnisse unterscheiden sich deutlich, je nachdem ob die Agenten auf eine vertrauenswürdige dritte Instanz zurückgreifen, oder ob sie nur ihre eigenen Transaktionsinformationen zur Einschätzung der Kooperationsbereitschaft potentieller Handelspartner heranziehen können. Bei allen Experimenten werden 3 Schreiner-Agenten eingesetzt, 2 kooperative (reputation $=1$ ) und 1 unkooperativer (reputation $=0$ ). Alle anderen Agenten sind kooperativ.

\subsection{Vertrauensunterstützung durch dezentrale Reputationsinformation}

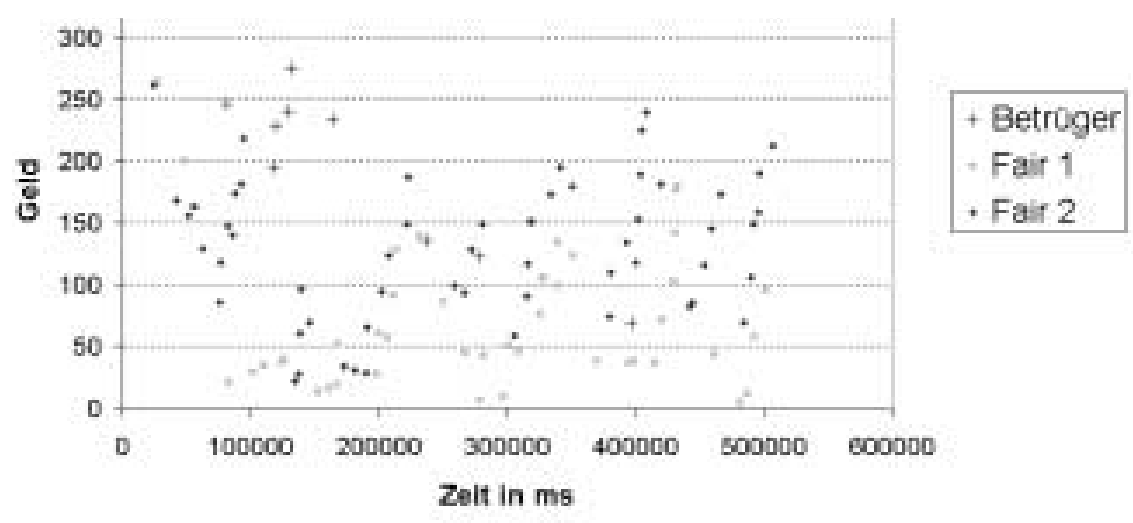

Abbildung 5: Nutzung eigener Reputationsinformationen.

In der ersten Testreihe nutzen die Agenten lediglich eigene Reputationsinformationen, die auf vorangegangenen Erfahrungen mit Handelspartnern beruhen. Bei unbekannten Agenten wird für die erstmalige Transaktion ein Reputationskoeffizient von 0.5 angenommen. Aus Abbildung 5 wird ersichtlich, dass der betrügende Agent zu Beginn der Testreihe erfolgreicher handeln kann als seine Mitbewerber. Im Lauf der Zeit wird er jedoch zunehmend vom Marktgeschehen ausgeschlossen, und die kooperativen Agenten führen vermehrt Transaktionen durch. Je mehr Transaktionen durchgeführt werden, umso mehr unterscheidet sich der Reputationskoeffizient der unkooperativen und der kooperativen Schreiner-Agenten. Die Rückwirkungen auf die Angebotspreise führen dazu, dass der unkooperative Agent nur $11 \%$ der Transaktionen durchführt. Der unkooperative Agent wird somit - allein durch seine ,schlechte“ Reputation - de facto vom Markt ausgeschlossen, wenngleich er in der Anfangsphase die Unkenntnis seiner Handelspartner erfolgreich ausnutzen kann. 


\subsection{Vertrauensunterstützung durch einen zentralen Reputationsdienst}

In der zweiten Testreihe wird eine vertrauenswürdige Instanz genutzt, die einen Austausch von Reputationsinformationen zwischen den einzelnen Agenten ermöglicht. Bei ansonsten gleicher Ausgangslage [vgl. Referenzszenario] zeigen mehrere Testläufe, dass der unkooperativ handelnde Agent sein Startkapitel nicht vermehren kann. Des weiteren führt er wesentlich weniger Transaktionen (10\%) als die kooperativen Agenten durch. Der unkooperative Agent wird somit de facto vom Marktgeschehen ausgeschlossen. Die kooperativ handelnden Agenten können hingegen ihre Kapitalbestände im Zeitablauf vermehren. Bedingt durch das geringere Risiko des gegenseitigen Vertrauens finden insgesamt mehr Transaktionen als bei der Nutzung einer dezentralen Reputationsinstanz statt, so dass der Markt insgesamt liquider und stabiler wird.

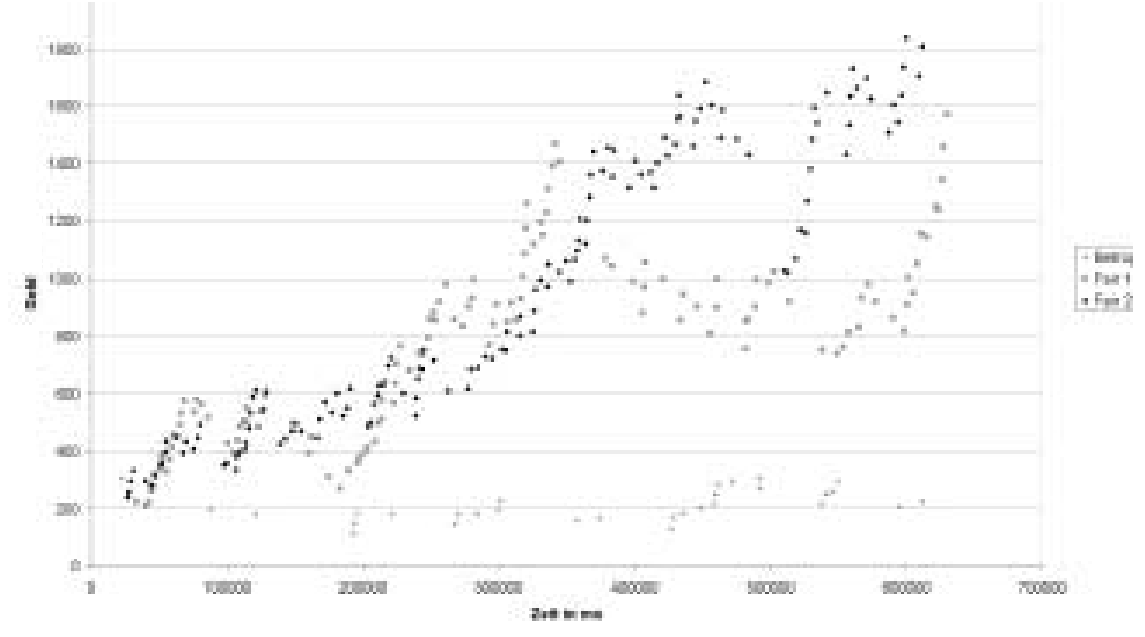

Abbildung 6: Entwicklung des Kapitals bei Nutzung einer zentralen Reputationsinstanz

\section{Zusammenfassung und Ausblick}

Um eine automatisierte Sanktionierung betrügerischen Verhaltens in offenen Systemen wie z.B. agentenbasierten elektronischen Märkten zu erreichen, muss es eine Rückkopplung von der Durchsetzungs- zur Anbahnungsphase geben, die Erfahrungen aus der Abwicklungsphase einer wirtschaftlichen Transaktion zur automatischen Modifikation von Auswahlentscheidungen in zukünftigen Informationsphasen nutzt. Es lässt sich zeigen, dass ohne den Einsatz eines solchen 
Reputationsmechanismus betrügerische Agenten erstens den größten Nutzen aus Transaktionen ziehen und zweitens die marktliche Koordinationsleistung des Marktplatzes zum Scheitern bringen.

In der vorliegenden Arbeit wurde für agentenbasierte elektronische Märkte ein Reputationsmechanismus konzipiert, im MAS AVALANCHE prototypisch implementiert, und die Ergebnisse einer zentralen und dezentralen Variante der Reputationsgenese einander gegenübergestellt. Das Verhalten der Agenten wird durch stochastische Elemente bestimmt, welches strategische Überlegungen simuliert. Durch den Reputationsmechanismus werden Risikoprämien eingeführt, so dass die Kaufs- und Verkaufsangebote der betrügerischen Agenten an Wettbewerbsfähigkeit verlieren. Daher führen die ehrlichen Agenten fast nur noch mit anderen ehrlichen Agenten Transaktionen durch; die betrügerischen Agenten werden im Laufe der Zeit vom Handel ausgeschlossen und das System insgesamt stabilisiert.

Eine dezentrale Realisierung zeigt erwartungsgemäß eine langsamere Sanktionierung betrügerischer Transaktionspartner. Obwohl explizit keine Sanktionsinstanz implementiert wurde, kommt es zu einer Relegation der betrügerischen Agenten. Die ehrlichen Agenten bilden untereinander ein Netzwerk des Vertrauens. Eine zentrale Realisierung durch eine dezidierte Reputationsinstanz bringt ein im Vergleich schnelleres Ergebnis, da die Information über die Betrügereigenschaft eines Agenten unmittelbar systemweit zur Verfügung gestellt wird. Die Wirkung der in einem offenen Marktplatz zunächst nur bilateral vorhandenen Reputationsinformation kann somit durch zentrale vertrauenschaffende Institutionen verbessert werden. Diese Variante geht allerdings von maximaler Vertrauenswürdigkeit der zentralen Instanz - im Interesse ihrer eigenen Reputation - wie auch der informationsbereitstellenden Agenten aus (einen Mechanismus zur Unterbindung der Verbreitung „falscher“ Reputationsinformationen stellt [Dela00] vor).

Zukünftige Experimente mit dem existierenden Prototyp sollen zeigen, welche Veränderungen sich unter Kosten- und Geschwindigkeitsgesichtspunkten durch die dezentrale Weitergabe von Reputationsinformation zwischen den Agenten, die Einführung von Zugriffskosten für die zentrale Reputationsinstanz oder den Einsatz anderer Bewertungsmechanismen [MoZa99] ergeben.

\section{Literatur}

[Dela00] Dellarocas, Chrysanthos: Immunizing Online Reputation Reporting Systems Against Unfair Ratings and Discriminatory Behavior. In: Proceedings of the Second ACM Conference on Electronic Commerce. ACM Press, Minneapolis, MN 2000.

[Eggs01] Eggs, Holger: Vertrauen im Electronic Commerce: Herausforderungen und Lösungsansätze. Universität Freiburg, 2001. 
[EgEn00] Eggs, Holger; Englert, Jürgen: Electronic Commerce Enquête 2000 - Vernetzte kleine und mittlere Unternehmen. Konradin-Verlag, Leinfelden-Echterdingen 2000.

[Eyma00] Eymann, T., (2000): Avalanche - ein agentenbasierter dezentraler Koordinationsmechanismus für elektronische Märkte. Dissertation, Albert-Ludwigs-Universität Freiburg, 2000. http://www.freidok.uni-freiburg.de/volltexte/147

[FrRe99] Friedman, E.J. und Resnick, P.: The Social Cost of Cheap Pseudonyms, Working Paper, School of Information, University of Michigan, Ann Arbor. http://www.si.umich.edu/ presnick/papers/identifiers/index.html, 1999

[HiRi79] Hirshleifer, J.; Riley, J.G.: The Analytics of Uncertainty and Information - An Expository Survey, Journal of Economic Literature XVII, 1979, S. 1375-1421

[HoU194] Hopcroft, J.E., Ullman, J.: Einführung in die Automatentheorie, formale Sprachen und Komplexitätstheorie. 3. korr. Aufl.. - Bonn : Addison-Wesley, 1994.

[KeHa00] Kephart, J.O., J.E. Hanson, and A.R. Greenwald: Dynamic Pricing by Software Agents. Computer Networks, 2000. http://www.research.ibm.com/infoecon/paps/ html/rudin/rudin.html.

[Klei97] Klein, D.B. (Hrsg.): Reputation: Studies in the voluntary elicitation of good conduct, Ann Arbor, 1997.

[LePa97] Lee, H.L., P. Padmanabhan and S. Whang: The Bullwhip Effect in Supply Chains, Sloan Management Review, 38, Spring, 1997, S. 93-102.

[MoZa99] Moukas, A., G. Zacharia and P. Maes: Amalthaea and Histos: MultiAgent Systems for WWW Sites and Reputation Recommendations, in Klusch, M. (Ed.), Intelligent information agents: agent-based information discovery and management on the Internet. Springer, Heidelberg, 1999.

[MüRa99] Müller, Günter; Rannenberg, Kai: Multilateral Security in Communications. Addison Wesley Longman, München 1999.

[MüSc97] Müller, Günter; Schoder, Detlef; Kohl, Ulrich: Unternehmenskommunikation: Telematiksysteme für vernetzte Unternehmen. Addison Wesley, Bonn 1997.

[Pado01] Padovan, Boris: Ein Vertrauens- und Reputationsmodell für Multi-Agenten Systeme. Dissertation, Universität Freiburg, 2001.

[Prei98] Preist, C., Economic Agents for Automated Trading, HP Technical Reports HPL98-77. Hewlett Packard Laboratories, Bristol, 1998.

[Ripp98] Ripperger, T.: Ökonomik des Vertrauens - Analyse eines Organisationsprinzips. Mohr Siebeck, Tübingen 1998.

[RoZ194] Rosenschein, Jeffrey S.; Zlotkin, Gilad: Rules of encounter - designing conventions for automated negotiation among computers. MIT Press, Cambridge 1994.

[Simo57] Simon, H. A.: Models of Man - Social and Rational. Wiley \& Sons, NY 1957.

[Will85] Williamson, O.E.: The Economic Institutions of Capitalism, Free Press, NY 1985.

[WoJe99] Wooldridge, M.J., Jennings, N.R.: Software Engineering with Agents: Pitfalls and Pratfalls. In IEEE Internet Computing, May/June 1999, Vol. 3, No. 3, S. 20-27. 